das nicht wie aus einer Weiherede für ein christliches Gotteshaus der Gegenivart? Wenn dann allerdings einige Zeilen weiter (Magn. 100b 7. $38 \mathrm{ff}$.) dic Verheißung ăfivov Eival nötig ist, um den Hausbesitzern und biedern Werkmcistern der Stadt Magnesia cs eindringlich zu machen, der Göttin zu Ehren Altäre vor ihren Türen zu errichten und sie sauber mit Tünche zu streichen (wobei den Widerwilligen mit der Aussicht

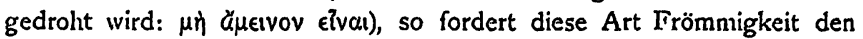
religiösen Menschen von heute notwendig zur Kritik auf. Und ebenso Magn. 98. Hier handelt es sich um die Verpflegung eines Opferstiers, der bis zum eigentlichen Festtage in freiwillige Obhut gegeben werden soll. Wer sich nun zu einer Beisteuer zu den Fütterungskosten versteht,

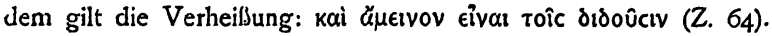

Dresden. (Lageado, Brasilien.)

G. Thieme.

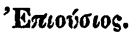

In G. Klein's gelehrter Abhandlung über die ursprüngliche Gestalt des Vaterunsers (I. Heft dieser Ztschr. 1906, S. 34ff.) ist, zumal es sich dabei um so tiefgreifende Untersuchungen wie das Verhältnis zur Johannestaufe, die Bitte um den h. Geist u. $\ddot{A}$. gehandelt, eine demgegenüber unbedeutende, überdies reichlich und überreichlich besprochene Einzelheit, nämlich die immer noch fragliche Bedeutung des Wortes $z$ tioúcıoc, unerörtert geblieben; nur indirekt konnte der Hinweis auf den Zusammenhang, in dem bei Mt die ersten drei, ja vier Bitten Erklärung und Begründung finden, wie v. 12 durch 14 . I5, so v. II durch 33.34 , dazu dienen, die ältere Erklärung (quotidianus, crastinus) $\mathrm{zu}$ beseitigen; und so ist überall dort die Bitte angeführt in der Form: "unser nötiges Brot". Näher gehen neuerdings auf diese Sache ein die Aufsätze von Lic. Dr. Gustav Hönnicke in der N. kirchl. Ztschr. v. 1906, wo S. 176 über jenes Wort so gehandelt wird, dab nach kurzer Erwähnung und Ablehnung anderer Deutungen die Erklärung Zahn's (z. d. St.) eingehende Besprechung findet. Wiedereintretend für die ältere Ableitung von $\hat{\eta}$ émıôc $\alpha$ sc. $\eta \dot{\eta} \mu \hat{\rho} \rho \alpha$ umschreibe derselbe die Bitte so: Gott möge uns heute das Brot für morgen geben, eine Erklärung, die durch Berufung auf Hieronymus, der im Hebräerevangelium an dieser Stelle מָּ gefunden, ferner auf einige Übersetzungen, wie die koptische, die crastinum, und die sahidische, die venientem übersetze, begründet werde. H. gibt zu, 
daß diese schon von andern wie Bengel, Meyer, Hofmann vertretene Erklärung viel für sich habe, während die Gegengründe teilweise nicht stichhaltig seien, z. B. daß einige Übersetzungen wie die syrische und die altlateinische Émıúcıoc auffassen im Sinne von fortwährend, beständig (quotidianus)(?), oder daß diese Erklärung einen Widerspruch mit Mt 6,34 , wonach Sorgen für morgen verboten sei, ergebe. Dennoch glaubt H. auf zwei Einwänden bestehen zu müssen, die er nicht so leicht wie

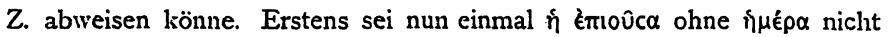
olne Weiteres Ausdruck für den morgenden Tag. Doch, um sogleich dies zu berichtigen, findet sich ja $T \hat{n}$ Ėmoúcn Act 20, 14; und wie oft $\hat{\eta}$

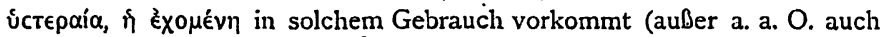
sonst wie bei Plato, Crito p. $44 \mathrm{~A}$ u. B), bedarf so wenig weiteren Nachweises wie die übliche Substantivierung von Adjektiven mit leicht ergänz-

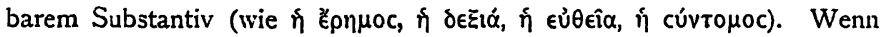
aber $\mathrm{H}$. fragt, warum denn, wenn es galt das aram. מָָ zu übersetzen,

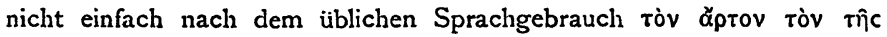

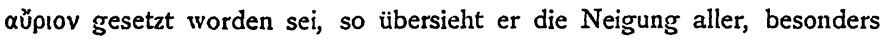
der alten Sprachen zur Abivechslung im Ausdruck, wie sich denn z. B.

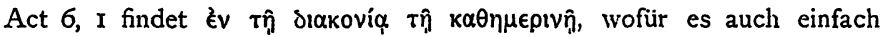

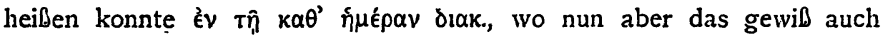

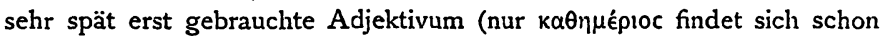
bei Klassikern, auch dieses höchst selten) gewählt wurde. Bedeutender ist der andere Einwand, daß der Gedanke, wie ihn $Z$. darstellt, logische Schwierigkeiten biete. Die Bitte: „gib uns täglich unser morgendes

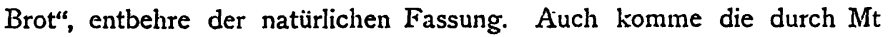
6, 34 abgewehrte Sorge doch dabei zum Ausdruck (womit also der vorher für nicht stichhaltig erklärte Einwand nachträglich anerkannt wird). $\mathrm{H}$. glaubt deshalb die Erklärung: „gib uns das uns angemessene, ausreichende Brot" vorziehen zu sollen, indem er émıov́cıoc von éteîval ableitet, wobei die Unterlassung der Elision in $E \pi \mathbf{i}$ vor dem Vokal am besten daraus zu erklären sei, daß die Wortbildung auf solche zurückgehe, die die griechische Sprache nicht völlig beherrschen. Über letzteres hatte sich schon früher C. Weizsäcker geäußert in den Untersuchungen über die evang. Geschichte, 1864, wo er (S. 407) übersetzt: „das notwendige Brot gib uns alle Tage" (ebenso in den Ausgg. des NT.s, so 1900 bei Mt 6, II. Lc II, 3 ,unser nötiges Brot"), und seine Ablehnung der gewöhnlichen Ableitung von $\dot{\eta}$ Ė $\pi$ iov̂ca mit Berufung auf den Kontext, nämlich den „ma@gebenden Gedanken“, so begründet: „die auffallende aber grammatisch mögliche Wortbildung aus $\dot{\epsilon} \pi \dot{i}$ und oủcía (wie $\dot{u} \pi$ - 
$\epsilon \xi o u ́ c 10 c)$ kann um so weniger von der sachlich richtigen Deutung abhalten, als das Wort unter die ganz freien Bildungen der NTlichen Sprache gehört", mit dem weiteren Zusatz: "je rascher das erste Verständnis verloren gchen konnte, desto leichter begreift sich, dab schon das Hebräerevangclium der Nazaräer nach Hieronymus mit רị übersetzte". Aus letzterem Satz, womit freilich die Sache auf den Kopf gestellt wird, als ob nämlich das griechische Vaterunser das Original wäre, das in das Hebräische oder Aramäische übersetzt worden sei, ist wenigstens zu sehen, daß die Notiz des Hieronymus (vollständig jetzt im Wortlaut bei E. Preuschen, Antilegomena, p. 5), an die nun Z. und Nestle (Einführung S. 112) erinnern, auch W. kannte, ohne sich jedoch dadurch in seiner Erklärung bestimmen zu lassen. Was aber die grammatische Möglichkeit betrifft, so hatte W. dieselbe zwar behauptet, jedoch nicht nachgewiesen. Diesen Mangel hat sich eifrig zu ergänzen bemüht Cremer in seinem bibl. theol. Wörterb. zur neutestamentl. Gräcität, wo dieser Punkt genau behandelt ist. Freilich so schlagende Beispiele wie Ėtılкท́c, Ėाоркєîv gibt es nicht sehr viele, und bei diesen erklärt sich der Mangel der Elision durch ursprüngliches Digamma; andere, bei denen diese Ėrklärung nicht zulässig, scheinen sich immerhin nur selten und weniger zuverlässige $z u$ finden. Richtig wird aber allerdings sein, daß solche Dinge wie Digamma und Hiatus in der späteren Gräcität weniger beachtet wurden, das Digamma nicht, indem wir n̉prácato finden $\mathrm{Mt}$ 25, 16. Mc 14, 6 (neben eipró́cato Mt 26, 10), der Hiatus aber, der schon der.Deutlichkeit wegen in der klassischen Gräcität vielfach nicht zu vermeiden war, jetzt wohl noch weniger Anstoß gab, als man sich aus Bequemlichkeit, um die feinere Unterscheidung zu ersparen, gewöhnt hatte das $v \in \notin \varphi \in \lambda \kappa$. überall, vor Konsonanten wie vor Vokalen, zu setzen (analog oủtwc, $\mu$ éxplc), wofür dann wohl auch umgekehrt ein Hiatus nicht als störend empfunden werden mochte. Großen Nachdruck aber legt Cremer mit Recht darauf, daß émioúcioc nicht von dem entfernteren émeîval (wie noch $\mathrm{H}$. tut) sondern (wie auch von W. geschehen) unmittelbar von dem nächsten, von oủcía, abgeleitet werde, sowie Ėzoúcioc nicht von é̉̇îval, sondern von oủcía (ein seines Vermögens, seiner oủcía, beraubter), und sucht weiter nachzuweisen, daß oủcí $\alpha$ nicht bloß Wesen (wie in $\delta \mu 00 v$ c1oc) sondern auch Existenz, Subsistenz bedeute, Ėrov́cioc also: zur Existenz nötig, ausreichend. Trotz dieser gründlichen Untersuchung schliebt aber Cr. mit der nicht volle Zuversicht verratenden Erinnerung, man dürfe nicht vergessen, daß es im geivöhnlichen Leben mit der Bildung der Wörter nicht so hergehe, wie es die Gelehrten nach 
ihren methodischen Gesetzen erwarten, nit andern Worten, dab man es so genau nicht nehmen dürfe. Als ob nicht gerade die Entwicklung der Volkssprache nach bestinmmten, man darf wohl sagen, Naturgesetzen vor sich ginge, von denen die am wenigsten Gebildeten unbedingt, obgleich unbewußt, geleitet werden! Jedoch wie steht nun die Sache? Die scheinbar nächstliegende Ableitung von $\hat{\eta} E$ emıûca hat unleugbar uniberwindliche Schwierigkeiten. Ungerechnet die Disharmonie von Mt 6, I I mit der, wenn auch etwa nicht ursprünglich, doch im Zusammenhang unseres jetzigen Textes bald folgenden Stelle 6, 34. ist die zweimalige Zeitbezeichnung in so kurzem Satz störend genug. Denn wenn auch unsere deutsche Bibel Lc II, 3 die anstößige Form (wörtlich: unser tägliches Brot gib uns täglich) durch die Übersetzung von $\kappa \alpha \theta^{\prime} \dot{\eta} \mu \dot{\epsilon}^{\prime} \rho \alpha v$ mit „immerdar" vermeidet, so daß man wahrscheinlich unlogischerweise auch am Urtext weniger Anstoß nahm, und wenn es bei Mt etwas erträglicher lautet: unser tägliches Brot - heute (cí $\mu \in p o v)$, wenn man ferner dieses notdürftig erklären kann: unser Brot für den kommenden Tag gib uns (schon) heute ( $d a ß$ wir uns nicht doch in den $z u$ fliehenden Sorgen verzehren), - das eine läbt sich nicht wegschaffen: die zweimalige Zeitbestimmung in so kleinem Satz bleibt eine Sonderbarkeit, für die man vergebens nach einer Erklärung sucht. Solche Bedenken müssen sich aber schon frühzeitig geltend gemacht haben, wie das seltsame Wort supersubstantialis beweist, das die Vulg. bietet Mt 6, I I (panem nostrum supersubstantialem), während sich für dasselbe Wort des Urtextes daselbst findet Lc II, 3: panem nostrum quotidianum; und zwar hat jenes Wort Hieronymus nach der oben erwähnten Notiz schon vorgefunden. ${ }^{x}$ Wie der Übersetzer oder Abschreiber, der die Bildung dieses sein griechisches Vorbild an Einzigartigkeit wo möglich übertreffenden Wortes gewagt, hierzu gekommen sein mag, läßt sich, wenn auch nicht nachweisen, doch vermuten. Entweder hatte und fand derselbe kein lateinisches Wort für das wiederzugebende griechische, dessen Bestandteile er zwar $z u$ erkennen glaubte, ohne sich aber über den Begriff klar zu sein, oder er fand schon die lateinische Übersetzung mit quotidianus (vielleicht auch crastinus) vor, ohne aber von deren Richtigkeit überzeugt zu sein, und suchte nun wie in einer Interlinearübersetzung die $\mathrm{hm}$ verständlichen Bestandteile, so gut er konnte, $\exists \pi i$ durch ein übergeschriebenes super und -oúcioc durch ein vermeintlich entsprechendes substantialis, nachzubilden. An eine Beibehaltung dieses Versuchs war aber schon wegen

I Pro supersubstantiali pane reperi mahar etc. a. a. 0 . 
der Unförmlichkeit und Unverständlichkeit des Ausdrucks nicht zu denken. Und nachdem IFieronymus sein mehr an der Ablcitung von $\dot{\eta} \ell$ triov̂cu zweifeln zu dưrfen. Ist nun aber cliese trotz allem nicht festzuhalten, so fragt sich, ob der andern Erklärung (von $e \pi i$ und oucía) unbedingt $z$ u vertrauen sein wird. Wenn auch dies nicht $z u$ behaupten ist, insofern sich immer wieder die Frage aufdrängt, ob nicht doch die Ableitung von ì émrov̂ca die näher liegende sei, so wird man, woran freilich schon von Anfang an zu denken war, fragen müssen, welches aramäische Wort denn wohl mit diesem Étıúcıoc wiedergegeben werden sollte. In den altjüdischen Gebeten, nach deren Muster, wic wir nach den neueren Untersuchungen wissen, das Vaterunser gebildet wurde, scheint man ein entsprechendes Wort noch nicht entdeckt zu haben. Franz Delitzsch hat nun in seinem hebräischen NT an beiden Stellen gesetzt: stimmten Teils oder Maßes, also das uns zukommende, beschiedene Brot, mit Anlehnung an Gen 47, 22. Prov 30, 8. Ezech 16, 27. Ergibt sich hierdurch unleugbar ein angemessener Sinn, so ist doch kaum wahrscheinlich zu machen, wie hieraus die Übersetzung mit émıúcioc entstehen konnte. Dennoch wird die Schwierigkeit nicht unlösbar sein, wenn man sich erinnert, da neben dem bekannten מุ cras, crastinus noch eine obgleich in der biblischen Sprache als solche nicht gebräuch-

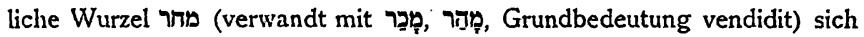
findet, wovon ein nicht eben seltenes Wort abgeleitet wird, רמ̣, Kaufpreis, merces, praemium laboris, Arbeitslohn, Hi 28, 15. Ps 44, 13. Jes 45, 13. 55, 1. Mi 3, Ir. 2 S 24, 24. Dt 23, 19; auch Dn Ir, 39: Ob nun dieses șִ selbst, nur defective geschrieben, oder eine andere Form desselben Stammes ursprünglich diese Stelle eingenommen, mögen Andere, die als Orientalisten hierin die erste Stimme haben, entscheiden. Ist unsere Vermutung begründet, so würde ein unbestreitbar passender Gedanke gewonnen: das Brot des Arbeitslohnes, somit unser verdientes, redlich erworbenes Brot, zu übersetzen also etwa: unser ehrliches Brot gib uns heute (täglich); zugleich aber wäre damit erklärt, was sonst immer ein Rätsel bliebe, wie schon in allerfrühester Zeit, als die Tradition noch von den ersten Jüngern her lebendig sein multe, bei diesen gewiß nie in Vergessenheit kommenden Worten die irrige Auffassung sich bilden konnte, die dann. bald in dem griechischen émioúcioc, lat. quotidianus ihren Ausdruck suchte. Mißverstand eines doppelsinnigen Wortes oder Wortstammes wiirde den seltsamen aber gerade bei solchen überall mit ehrfurchtvoller Scheu gesprochenen und gehörten 
Worten verzeihlichen und bei der unendlichen, daher wohl oft gedankenlosen, Wiederholung lange unbemerkt gebliebenen Irrtum verschuldet haben.

Weißenburg $\mathrm{i} / \mathrm{B}$.

A. Bischoff.

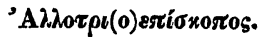

Wenn auch die Bestandteile des in der ganzen Gräcität nicht mehr und im NT an der einzigen Stelle I Petr 4, I5 vorkommenden Wortes,

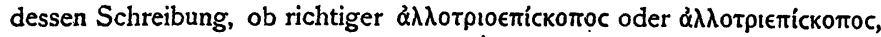
für jetzt dahingestellt bleibe, offen vorliegen, scheint doch die Bedeutung desselben für unsere Stelle, um die es sich ja allein handeln kann, wenigstens nicht allgemein richtig erkannt zu werden, soweit sich nämlich ohne Kenntnis aller einzelnen Kommentare aus den am meisten gebrauchten Übersetzungen urteilen läßt, Vulg.: „alienorum appetitor", L.: "der in ein fremd Amt greift", H. A. Schott: "male sedulus", Weizsäcker: "der sich fremder Dinge anmaBt". Cremer verweist in seinem Lexikon s. v. auf Plato's Phaedrus p. 230 A. (genauer p. 229 E.), wo sich aber nur die Äußerung des Sokrates findet, er halte für lächerlich sich um fremde Dinge zu kümmern, ehe man die nach dem Delphischen Spruch

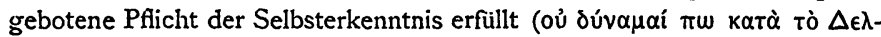

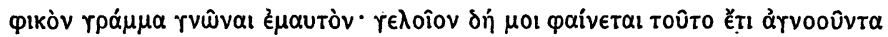

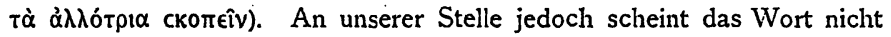
blob wie in der platonischen unnütze und gegenüber der weit wichtigeren Selbsterkenntnis unbedeutende Beschäftigung, wie dem Sokrates dort die mythologischen Fragen über Boreas, Chimaira, Hippocentauren und Pegasus erscheinen, sondern ein höchst bedenkliches und streng $\mathrm{zu}$ meidendes Tun zu bedeuten, vor dem mit allem Ernst zu warnen sei. Nach der gewöhnlichen Ansicht wäre ein Verhalten gemeint, das an sich gewiß tadelnswert leicht auch schädlich wirken, aber kaum als so gefährlich gelten kann, um in diesem Zusammenhang genannt zu werden. Cremer verwirft daher Luther's Auffassung, hält aber selbst dafür, daß die Sünden wider $\dot{d}$ as achte Gebot gemeint seien, ohne jedoch diese Ansicht näher zu begründen. Die Möglichkeit dieser Beziehung ist nun freilich dem Wortlaut nach nicht zu bestreiten, insofern z. B. ein $k \alpha \tau \alpha-$

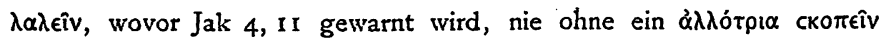
wird entstehen können; aber für unsere Stelle ist diese Bedeutung nicht ausreichend. Denn der Zusammenhang ist ja dieser, daß die Leser ermahnt werden, in drohenden Leiden nicht $\mathrm{zu}$ verzagen, insbesondere 
nicht, wcnn sie um Christi willen geschmäht werden. Denn nur in diesem Fall, so will das ráp des Úberganges v. 15 sagen, wenn ihr um Christi willen Schmach leidet, seid ihr Makaptot, nicht aber, wenn ihr selbst Unglück verschuldet, also verdiente Strafe erfahret, sondern nur wenn als Christen usiv. (v. IG). Wenn es also hier heibt: keiner von euch leide ẃc povev̀c $\hbar k \lambda \epsilon \pi \tau \eta \bar{c}$, so sieht man, daß hiermit auf Handlungen hingewiesen ist, die vom bürgerlichen Strafgesetz betroffen sind, deren kein Mensch, wieviel weniger ein Christ, sich schuldig machen soll. Auffallen kann hier, um dics beiläufig zu bemerken, dab als drittes каколою́c gesetzt ist, das sonst z. B. oben (I Petr) 2, 12 allgemein: Übeltäter, Missetäter bedeutet. Doch darf man, wenn diese Nebenbemerkung gestattet ist, annehmen, daß dieses Wort hier spezielle Bedeutung hat, wie etwa lat. malus bei Hor. sat. I, 4, 3: si quis erat dignus describi quod malus ac fur, wo malus einen Schelm oder Schurken bedeutet, dessen Tun, wenn auch nicht eigentlich Diebstahl, doch (durch Betrug, Unterschlagung, Veruntreuung u. dgl.) nicht minder gefährlich, daher ebenso von den Gesetzen bedroht ist, ib. I, 3, 59: nullique malo latus obdit apertum (wo malo masc., nicht neutr., weil nur so das Bild passend: latus obdit apertum), besonders noch ib. I, 1,77: formidare malos, fures (nicht malos fures, böse Diebe, sondern:) Schelme, Diebe, weil die Angst des reichen Filzes desto besser veranschaulicht wird, je mehr Feinde er fürchtet. $\mathrm{Zu}$ vergleichen ist hier auch der Ausdruck: „Zöllner und Sünder", wo das Wort „Sünder" gleichfalls in einem speziellen Sinn (im Gegensatz zu den Zöllnern, die ja von den Sündern im allgemeinen nicht ausgenommen

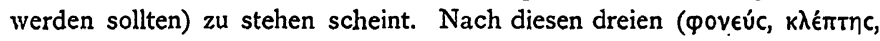

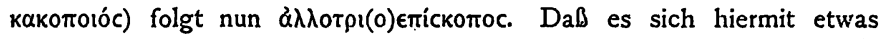
anders verhalten wird, ist schon aus der Wiederholung des wc zu sehen. .Um so gemeine Verbrechen wie Mord, Diebstahl, Betrug scheint es sich nicht zu handeln, da sonst das Wort einfach jenen an die Seite gestellt werden konnte, immerhin aber auch um Handlungen, die zum Konflikt mit dem Strafgesetz führen mußten. Ein Übergreifen in fremdes Amt, überhaupt in fremde Angelegènheiten, war aber vom geltenden römischen Strafgesetz so wenig bedroht wie üble Nachrede gegen den Nächsten, wenn durch solche nicht geradezu. die Wohlfahrt des Andern gefährdet wurde. Als strafbar nun bleiben, nachdem der Vergehen und Verbrechen gegen Leben und Eigentum.der Mitbürger schon gedacht ist, nur übrig politische Handlungen, denen eine staatsgefährliche Absicht zugeschrieben werden konnte. Alle solche Handlungen fielen unter die lex maiestatis, wonach ein crimen minutae oder laesae maiestatis in der Zeit der Republik 
zwạr nur durch wirklichen Vaterlandsverrat, später aber in der Kaiserzeit mehr und melir schon durch Worte und Mienen, überhaupt durch die harmlosesten Schritte begangen werden konnte, wie aus Tacitus und Sueton sattsam bekannt ist, wobei freilich zu bemerken, da $\mathrm{im}$ allgemeinen mehr Angehörige vornehmen als geringen Standes deshalb verdächtigt und verurteilt wurden. Die Gefahr solcher Anklagen wurde aber immer größer, je mehr die öffentlichen Zustände zu Beschwerden Anlaß gaben, und gerade fromme Gläubige mochten, nicht in eigenem Interesse wohl aber in dem ungliucklicher Mitbürger, an Maßregeln der Obrigkeit oft berechtigten Anstoß nehmen. $U m$ s.o eindringlicher wird in den neutestamentlichen Briefen vor, allen möglicherweise verdachterregenden Schritten gewarnt und Gehorsam gegen die bestehende Obrigkeit eingeschärft. So hier oben (I Petr): 2, 13. I7ff. Röm 13, 1-7. I Tim 2, 2. Tit 3, r. Ist hier, wie somit anzunelimen, auf solches hingedeutet, so ist auch leicht $\mathrm{zu}$ sehen, weshalb der etwas euphemistische Ausdruck gewählt ist. Denn an sich bedeutet das Wort freilich nicht homo rebellis, nicht einmal insidiator, sondern synonym mit katáckoroc nur allgemein: explorator, Späher, wie übrigens auch Ėंíckoпoc selbst gebraucht ist

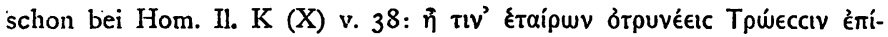
скопоv; willst du einen der Genossen (zu) den Troern als Späher senden? Aber die Leser des Briefes konnten sicherlich verstehen, was das Wort hier bedeute, da für alles ob auch mißgünstige Einmischen in fremde Dinge oder gar bloßes Beobachten und Beurteilen der Angelegenheiten anderer, wenn் es nur Privatsachen betraf, keine gerichtliche Strafe zu fürchten war. Immerhin schien die Unbestimmtheit des Ausdruckes gegen den Verdacht zu schützen, als ob der Verfasser annehme, daß staatsgefährliche Absichten oder nur Gesinnungen unter den Gläubigen gehegt würden.

Es soll aber nicht verschwiegen werden, daß eine nahezu übereinstimmende Auffassung der Stelle sich schon bei Bengel findet, der sich so darüber äußert: Tales sunt qui sese in negotia publica aut privata, sacra-aut civilia, ad ipsos nil pertinentia, quasi magna prudentia et fidelitate et odio mundanae iniquitatis impellerentur, ingerunt. cuius modi homines . . facile in "passiopes" incurrunt. Man sieht, daß B. den $\mathrm{Zu}$ -

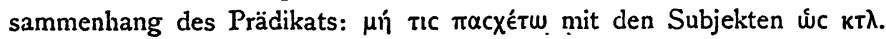
nicht verkennt, also auch die Gefahr schwerer gerichtlicher Bestrafung angedeutct findet; ${ }^{x}$ wenn er nur nicht die negotia privata geglaubt hätte

1 Wie er auch noch bemerkt: idque imprimis fieri poterat apud ethnicos magistratus. Zeitschr. L. d. neutest. Wiss. Jahrg. VIl. 1906. 
nit herbeizichen zu sollen. So mögen wohl auch andere Ausleger die richtige Auffassung vertreten. Meistens scheint jedoch das neugierige und übelwollende Beobachten und Besprechen fremder Privatsachen inmer noch zu sehr hereingedcutet zu werden, wodurch diese Warnung

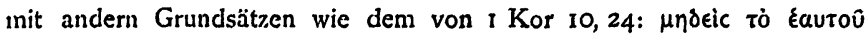

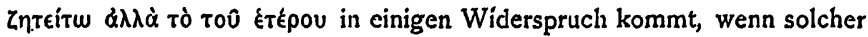
nicht mehr oder weniger künstlich verwischt wird. - Es ist aber auch noch hinzuweisen auf die Fortsetzung im Text, wodurch die obige Erklärung bestätigt wird. Wenn es heißt v. 16: „leidet er aber als Christ, so schäme er sich nicht usw.", so sind, da ja mácxet aus v. 15 zu ergänzen, nicht Nachteile und Anfechtungen im Privatleben, wie sie wohl einen Religionswechsel schon damals begleiten mochten, sondern gerichtliche Strafen gemeint, wie sie Mörder und Diebe treffen, von denen aber auch Bekenner des Christenglaubens selbst unter dem milden Regiment eines Trajan nach dessen bekanntem Brief an Plinius (ep. X, 97 (98), wenn sie nicht tätige Reue bewiesen (supplicando diis nöstris), bedroht

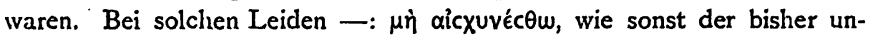
bescholtene Mann es als Schmach empfinden muß, mit den staatlichen

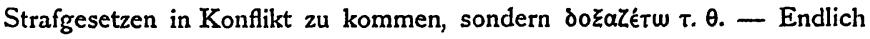
sei noch die Bemerkung gestattet, daß die soeben geäußerte Vermutung, es möchten auch andere zum richtigen Verständnis des Wortes gekommen sein, sich schon bestätigt hat, indem der, wie angedeutet, nicht über umfassende Kenntnis der neueren exegetischen Literatur verfügende Verfasser leider erst jetzt bei Gunkel (d. Schr. d. NT.'s neu überrs. usw. z. d. St.) die Anmerkung findet: „Aufruhrer, novarum rerum cupidus (Deißmann).“ Wenn aber diese Auffassung doch nur als "möglich" erwähnt, neben und vor dieser aber die gewöhnliche vorgetragen wird (wonach z. B. auf taktlose Zudringlichkeit von Wanderpredigern angespielt sei, als wäre auch solches Verhalten von gerichtlicher Strafe bedroht gewesen), so wird der vorstehenden Besprechung auch jetzt noch Berechtigung nicht abzusprechen sein.

Weißenburg $\mathrm{i} / \mathrm{B}$.

A. Bischoff.

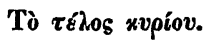

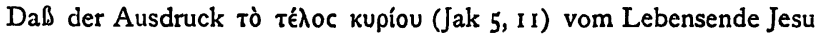
verstanden werden könne, soll nach $Z$ ahn (Einl. I $\left.{ }^{2}, 54\right)$ schon deshalb unmöglich sein, weil neben $\delta^{\circ}$ кúproc, das in demselben Vers zweifellos Gott bezeichne, das artikellose kúproc am wenigsten auf. Jesum sich beziehen könne. Wo eine Unterscheidung stattfinde, sei umgekehrt kúproc Gott, $\delta$ kúploc Jesus, so Jak 5, 8. 10. 14. 15. Aber schon die beiden letzten 
Stellen beweisen nicht, was sie sollen. Entweder wird den Kranken derselbe gesund machen, aufrichten ('́repeî aủròv $\delta$ K.), in dessen Namen ( $\varepsilon v$ óvó $\mu a r ı$ K.) Gebet und Ölsalbung angewendet worden, oder es wird der, für den im Namen Jesu gebetet worden, durch Gottes Hilfe genesen, nicht aber umgekehrt. Auch oben I, I hieß cs: $\theta$ Eov̂ kai kupiou

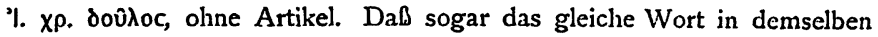
Satz verschiedene Subjekte bezeichnen kann, beweist das bekannte Beispiel, wo im Gegensatz zum Grundtext, der nicht dasselbe Wort wiederholt (Ps I IO, I : 'יהצֶ), die Übersetzung, wwie absichtlich änigmatisch dasselbe Wort in verschiedener Bedeutung gebraucht: eimev

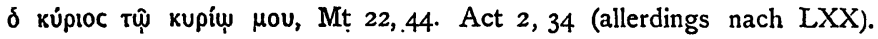
Umgekehrt kann man sagen, dals kupíou dem 'lẃß des vorausgehenden Satzes entsprechend als nom. propr." und deshalb olne Artikel stehe, dagegen am Ende des Wortes $\delta$ xúptoc als nom. appell., daher mit Artikel (gleichsam: „der Herrgott"). Es ist jedoch der Gebrauch des Artikels in solcher Verbindung überall so schwankend, daß hierauf allein ein sicherer Beweis nicht zu gründen ist. Wörtlich heißt nun jedenfalls

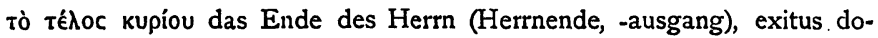
mini. Hier darf wohl gefragt werden, ob es denn, wenn auch die Worte

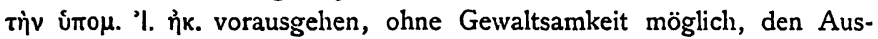

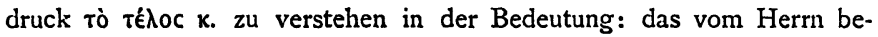
wirkte Ende - wessen? Hiob's? unmöglich; denn er lebte ja ,nach diesem" (Hi 42, 16) noch 140 Jahre, ein Zeitraum, der doch nicht einfach als in $\tau$ é̉oc inbegriffen zu denken wäre; noch weniger äber kann es heißen sollen: das Ende der allerdings soeben genannten, daher für

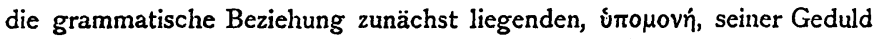
(als ob diese den Vielgeprüften doch endlich verlassen hätte! womit sich

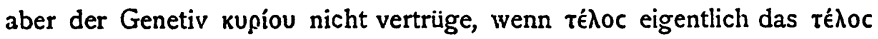

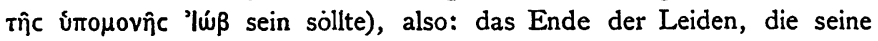
úropovń so lange getragen; denn so muß man ja dann erklären: das

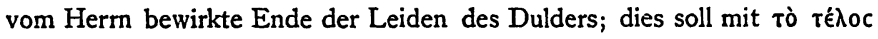
к. (Herrnende) bezeichnet sein. Eine für den schlichten Leser etwas starke Zumutung, mit dem einfachen Ausdrucke "des Herrn Ende (Ausgang)" einen so verschiedenes enthaltenden Begriff zu verbinden. Aber, so wird eingewendet, kann denn der Verfasser seine Leser an Jesu Lebensende erinnern wollen? Ohne allen $Z_{w e i f e l ;}$ wenn man nur, was sich doch von selbst verstehen sollte, dieses Lebensende auf Leiden und Sterben des Gekreuzigten beschränkt und nicht auch Auferstehung und Himmelfahrt darunter verstehen zu müssen meint, wobei man dann nicht 
stehen bleiben könnte, ohne auch noch dic Erhöhung \%u himmlischer

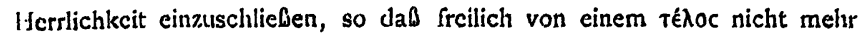
dic Rcde sein könntc. Ist es also, auch für gläubigen Standpunkt, nicht unzuliassig, von einem Lebensencle des Herrn in diesem Sinn, mit der Buschränkung auf Leiden und Sterben, zu sprechen, so ist nur die Frage, ob die Lescr daran als an etwas, das sie selbst gesehen, erinnert werden konnten. Aber Augenzeugen konnten, wenn auch frcilich nicht alle, welches anzunehmen nicht nötig, so doch manche Leser gewesen sein, zumal wenn, wie ja vielc wollen, dieser Brief eine der ersten oder gar die älteste Schrift des NT.'s ist; im anderen Fall, wenn sie späteren Ursprungs ist, paliste solche Voraussetzung trefflich zur Fiktion der Autor-

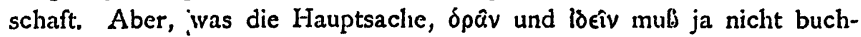
stäblich Augenzeugschaft bedeuten, sondern kann dasselbe sein wie

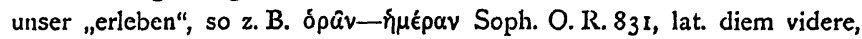
Tac. Ann. I, 8. Jedoch, so hören wir weiter, die gewöhnliche Verbindung

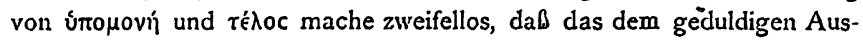
harren Hiob's entsprechende segen- und ruhmgekrönte Ende dieser Leiden gemeint sei. Aber die zu vergleichenden Stellen (Mt IO, 22. 24, 13. Jak 1, 4) sind anderer Art. Wo vom Beharren bis ans Ende die Rede, bedeutct réloc den äußersten, letzten Grad des Leidens, worin die Geduld sich bewähren mub, wenn nicht die ganze Prüfung vergeblich gewesen sein soll; hier müßte es die Befreiung und herrliche Belohnung bedeuten. Denn wenn es heißt: $\delta$ útroutivac eic $\tau$. oûtoc -, so ist

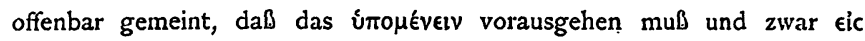
Té̉oc, dann erst tritt ein, was bezeichnet wird mit oũtoc cw $\theta$. Ebenso

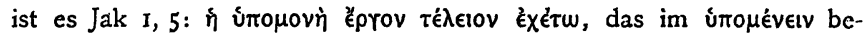
stehende êprov muß bis zur letzten Bewährung geleistet, muß ein té $\lambda \in i o v$ sein, dann erst ist $z u$ erwarten, was v. 12 sagt: - $\mu \alpha$ ḱ́ $\lambda \eta \dot{\mu} \mu \epsilon \epsilon \alpha_{1}$ - Aber freilich das Haupthindernis, das die Annahme der einfachsten Erklärung erschwert, ist noch ein anderes, das Bedenken nämlich, daß dadurch das Beispiel Jesu demjenigen Hiob's an die Seite gestellt werde, Christus und Hiob auf eine Stufe! Jedoch ist dies ja nur eine Seite, woraus keineswegs eine allgemeine Gleichstellung gefolgert werden dürfte. Wie? hat doch Christus selbst die Nachfolge, also die Befolgung seines Beispiels, in der Kirche imitatio genannt, den Seinigen empfohlen, Mt 10, 38. '16, 24 u. Parall., Anweisungen, die in der apostolischen Zeit fortgesetzt wurden, wie I Petr 2, 2I, wo Christus den

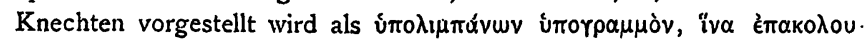

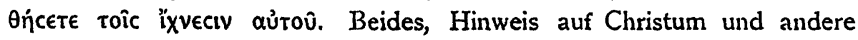


Vorbilder, findet sich verbunden in Hebräerbrief 12, 2. 3: "lasset uns (laufen durch Geduld und) aufsehen auf Jesum, den Anfänger und Vollender des Glaubens, und 13, 7: gedenket an eure Lehrer, ihr Ende schauet an und folget ilrem Glauben nach", worauf sich dann unmittelbar anschließt: "Jesus Christus gestern und heute usiv." Wenn nun an unserer Stelle bei der Ermahnung zur Geduld nach den Hinweisen auf die Propheten und auf Hiob der Gedanke an das höchste aller hiefür vorzustellender Vorbilder dem Verfasser sich aufdrängte, . kann dies so wenig anstößig sein, daß es, wenn es fehlen würde, schmerzlich vermil werden müßte. Außerdem könnte noch erinnert werden, daß der Satz

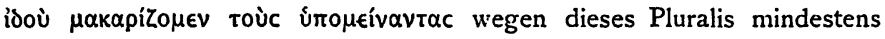
zwei Beispiele erwarten läßt. Denn unerträglich künstlich würde es sein, da doch gewiß das folgende

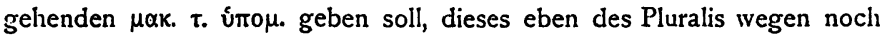
auf die vorhergenannten Propheten zurückbeziehen zu sollen. Auf diesen Grund würde zwar zu verzichten sein, wenn sich, wie noch $z u$ bemerken, eine Änderung der Textesgestalt empfehlen sollte, während bei Festhaltung des überlieferten Textzusammenhanges dieses Argument sein volles. Gewicht behält. Eines aber wird man nicht der vertretenen Auffassung entgegenhalten, daß nämlich eine Erwähnung der dem Hiob zuletzt gewordenen Belohnung fehlen würde, da auch bei den Propheten eine solche nicht genannt ist, überhaupt nicht zu nennen war, insofern der Entschluf, Heroen wie die Propheten und Hiob zum Vorbild zu nelimen, abgesehen von jeder zu hoffenden Belohnung an sich schon durch den Reiz solchen Vornelımens hinreichend begründet ist. Zudem

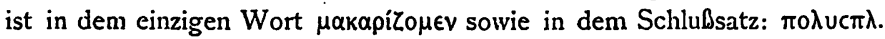
$\epsilon$. $\delta$ к. к. oikr. die Aussicht auf künftige Herrlichkeit genügend angedeutet. - Bis hierher hatte die Erklärung unserer Stelle keine besondere Schwierigkeit. Bedenken aber erregt der Schlufjsatz und dessen Zusammenhang mit dem Vorhergehenden. Auch von jenen, die mit

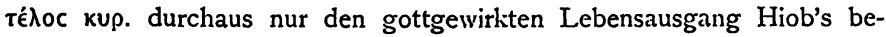
zeichnet wissen wollen, haben doch manche, unter ihnen Hofmann, dem

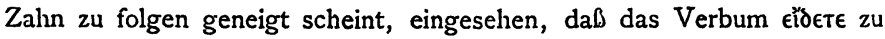
solchem Objekt nicht paßt, da ja in diesem Fall das zweite (Hiob's Ausgang) ebenso wie das erste (sein geduldiges Ausharren) nur durch Tradition erfahren, also gehört, nicht gesehen und selbsterlebt heißen könne; um jedoch durchaus der einfachsten Erklärung vom Lebensende Jesu auszuweichen, flüchtete man zu dem Vorschlag ỉ $\delta \in t \epsilon ~ z u$ lesen mit vorausgehender starker Interpunktion: von Hiob's Geduld habt ihr 
gehört und von seinem gottgewirkten Lebensausgang; sehet (imper.), daf, Gott gnädig ist und barmhcrzig (nötigenfalls auch bei der I.A. eǐdere anzutvenden: ihr habt (also daran) gesehen, daß Gott gnädig ist und

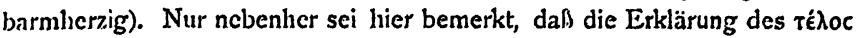
к. vom Lebensende Jesu auch mit dieser Konstruktion bcibehalten werden

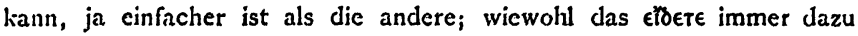
aufforderte, es auf das nähcr liegende und selbsterlcbte Ereignis zu bezichen, wie es denn auch $z u \mathrm{dem}$ auf die in grauer Vorzeit liegende Geschichte bezüglichon j̉Koúcate den treffendsten Gegensatz bildet. Schwcr annehmbar ist aber jene Konstruktion Hofmann's wegen der Unwahrscheinlichkeit der Verbindung eines Verbum's mit zwei Objekten, in deren Mitte es stände, statt wie gewöhnlich vor oder hinter beiden, wo nicht der Grund so auffallender Stellung leicht zu erkennen (anders

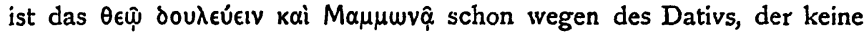
so direkte Beziehung enthält wie der Akkusativ), dann auch wegen der Verbindungslosigkeit, womit, ohne jede den Zusammenhang andeutende

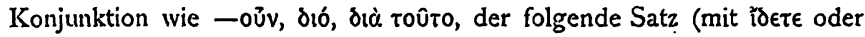

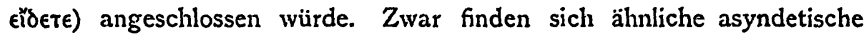
Strukturen auch sonst in Schlußformen, oben (Jak) 2, 22.24 $\beta \lambda$ é $\pi \epsilon 1 C-$, dann ¿́pâte, doch geht beidemale eine andere (die 3.) Person voraus, wodurch

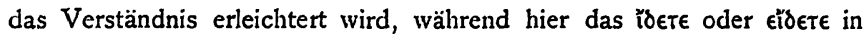
ganz andersartiger Bedeutung und Struktur gebraucht würde als das

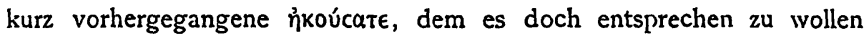

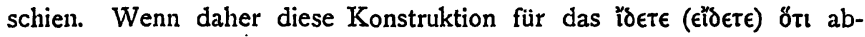
zulehnen ist, so bleiben mehrere Möglichkeiten. Entweder bringt der

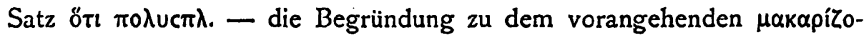
$\mu \epsilon \nu$, wobei die $Z$ wischensätze: wären: wir preisen selig die Dulder (wie ihr ja von Hiob's Geduld gehört und des Herrn Ende selbst erlebt), denn (oder: weil) der Herr gnädig ist und barmherzig. Beispiellos ist solche Verbindung nicht, s.

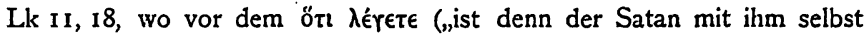
uneins? dieweil ihr saget usw.?) notwendig zu ergänzen ist: eine so ungereimte Meinung scheint ihr $\mathrm{zu}$ hegen, oder ähnliches. So wäre hier

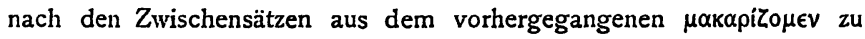
ergänzen: ja, selig sind sie gewiß (oder ähnlich), denn - Ebenso ist es Lk 20, 19 mit ěrvwcav ráp, womit nicht der Grund angegeben sein

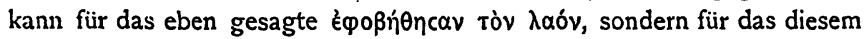

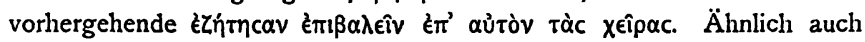

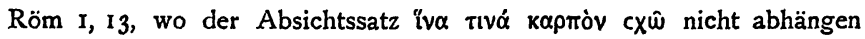




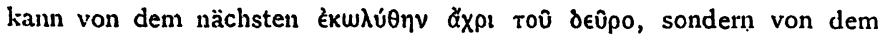

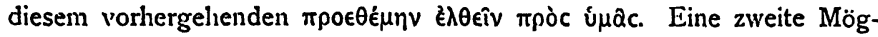
lichkeit zur Herstellung des Zusammenhanges wäre gegeben, wenn man

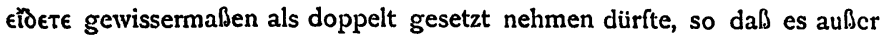
seinem Akkusativ noch einen Satz mit ö $\tau \imath$ zum Objekt hätte: des Herrn Ende habt ihr gesehen (und somit, da euch ja seine Erhöliung bekannt, auch gesehen) daß Gott usw. Auch solche Verbindung, ob man nun dabei von Brachylogie (Breviloquenz) oder von Prägnanz spreche, würcle kaum beispiellos sein. Etwas ähnlich sind die bekannten Fälle, wo das Subjelt des regierten Satzes als Objekt des Hauptsátzes antizipiert wird,

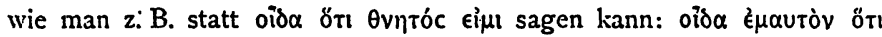
$\theta v$. $\epsilon$, insofern auch hier von demselben Verbum ein Akkusativ und noch ein Satz mit $8 \pi \imath$ abhängt. Endlich wäre noch zu helfen durch

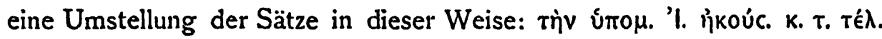

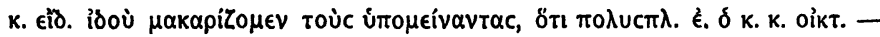
Es wird gestattet sein, hier zunächst nur auf diese drei Wege zur Herstellung des zugestandenermaßen schwierigen Zusammenhanges hinzuweisen, ohne schon einem derselben den Vorzug zu geben, da der Nachweis für die Richtigkeit des einen sowie der notwendig damit zu verbindende von der Ungangbarkeit der beiden andern für sich langwierige grammatische oder stilistische, wohl auch kritische, Untersuchungen erfordern würde. Wie übrigens das Urteil über diesen letzten Punkt ausfallen

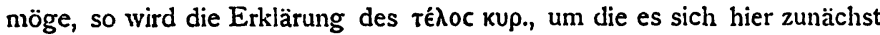
gehandelt, genug gesichert sein, um nicht durch jene Entscheidung auf's neue in Frage gestellt zu werden.

Weißenburg $\mathrm{i} / \mathrm{B}$.

A. Bischoff.

\section{Zum neutestamentlichen Griechisch.}

Gegenwärtig herrscht vielfach das Bestreben, die semitischen Einflüsse auf das biblische Griechisch möglichst gering anzuschlagen, am Ende ganz abzuleugnen. Wie kann man aber ohne solche den Sprachgebrauch erklären, der Mt 10; 32 und Lc 12, 8 vorliegt? Mit Recht bemerkt Zahn

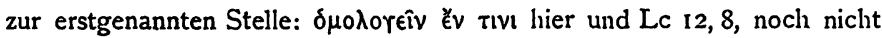
in LXX, weil hebr. הוֹ (welchem $\delta \mu 0 \lambda . . .$. entspricht), im AT nicht mit $I$ konstruiert vorkommt; wohl aber das entsprechende jüdisch-aram. 'Ṭ und christlich-syr.

Auch Blaß (Gr. $\$ 42,2)$ bezeichnet die Konstruktion mit Recht als einen Syrismus. Wie fremd sie einem Griechen war, zeigt die Tatsache, 